\title{
A Concise Asymmetric Synthesis of cis-2,6-Disubstituted $N$-Aryl Piperazines via Pd-Catalyzed Carboamination Reactions
}

\author{
Josephine S. Nakhla and John P. Wolfe* \\ Department of Chemistry, University of Michigan, 930 N. University Avenue, Ann Arbor, Michigan
} 48109-1055

\section{Supporting Information}

Experimental procedures and characterization data for new compounds in Tables 1-2 and Schemes 2-3 (100 pages).

General: All reactions were carried out under an argon or nitrogen atmosphere in oven- or flame-dried glassware. All catalysts, reagents, and aryl bromides were obtained from commercial sources and were used without further purification. $N$-phenyl-L-phenylalanine and $N$-phenyl-L-valine were prepared according to published procedures. ${ }^{1}$ DEPBT was prepared according to the procedure of Goodman and was purified by recrystallization from petroleum ether:ethyl acetate (1:1) followed by trituration with ethyl acetate to yield a white solid. Use of pure, colorless, reagent was essential to prevent degradation of enantiomeric purity during amide bond formation. Toluene, THF, ether, and dichloromethane were dried and purified using a GlassContour solvent purification system. Structural and stereochemical assignments were made on the basis of 2-D COSY, HSQC, and NOESY experiments. Ratios of diastereomers were determined by ${ }^{1} \mathrm{H}$ NMR and/or capillary GC analysis of crude reaction mixtures. Yields refer to isolated yields of compounds estimated to be $\geq 95 \%$ pure as determined by ${ }^{1} \mathrm{H}$ NMR, GC, and/or combustion analysis. The yields reported in the supporting information describe the result of a single experiment, whereas the yields reported in Tables 1-2 and Schemes 2-3 are average yields of two or more experiments. Thus, the yields reported in the supporting information may differ from those shown in Tables 1-2 and Schemes 2-3. 


\section{Synthesis of Substrates (Schemes 2-3)}

General Procedure 1: Conversion of $N$-Phenyl Amino Acids (2) to $N$-Allyl- $N$-Benzyl- $N$ '-Phenyl Amino Amides (3). A flame-dried round-bottomed flask equipped with a magnetic stirbar was cooled under a stream of nitrogen and charged with the $N$-phenyl amino acid substrate (1.0 equiv). THF was added to provide a $0.5 \mathrm{M}$ solution, which was cooled to $0{ }^{\circ} \mathrm{C}$ and stirred. DEPBT ${ }^{2}$ (1.2 equiv) was added, followed immediately by $N$-benzylallylamine (1 equiv), and the resulting reaction mixture was stirred at $0{ }^{\circ} \mathrm{C}$ until the starting amine had been consumed as judged by crude ${ }^{1} \mathrm{H}$ NMR analysis of an aliquot (ca. 3-4 h). Aqueous sodium bicarbonate was then added at $0{ }^{\circ} \mathrm{C}$, and the resulting yellow reaction mixture was extracted with ethyl acetate. The combined organic layers were washed with brine, dried over anhydrous sodium sulfate, filtered, and concentrated in vacuo. The crude product was then purified via flash chromatography on silica gel.

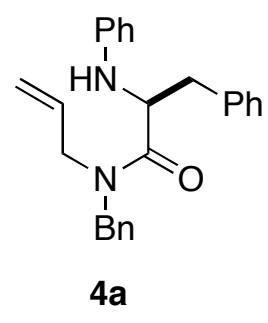

(S)- $N$-Allyl- $N$-benzyl-3-phenyl-2-phenylaminopropionamide $\quad \mathbf{( 4 a ) . ~ G e n e r a l ~ p r o c e d u r e ~} 1$ was employed for the coupling of (S)-3-phenyl-2-phenylaminopropionic acid ${ }^{1}$ (3.63 g, $\left.15.0 \mathrm{mmol}\right)$ with $N$ benzylallylamine $(2.21 \mathrm{~g}, 15.0 \mathrm{mmol})$. This procedure afforded $3.79 \mathrm{~g}(68 \%)$ of the title compound as a yellow oil. The enantiopurity was judged to be $98 \%$ ee by chiral hplc analysis (chiralcel OJ-H, 10\% isopropanol/hexanes, $1 \mathrm{~mL} / \mathrm{min}, \mathrm{RT}=11.14 \mathrm{~min}$ and $18.44 \mathrm{~min}$ ). This molecule was observed as a 1.5:1 mixture of rotamers; data are for the mixture. ${ }^{1} \mathrm{H} \mathrm{NMR}\left(400 \mathrm{MHz}, \mathrm{CDCl}_{3}\right) \delta 7.31-7.17(\mathrm{~m}, 9 \mathrm{H})$, 7.14-7.09 (m, 2 H), 6.92-6.91 (m, $1 \mathrm{H}), 6.77-6.71(\mathrm{~m}, 1 \mathrm{H}), 6.66$ (d, J = 8.0 Hz, $1 \mathrm{H}), 6.54(\mathrm{~d}, J=7.5$ Hz, 1 H), 5.73-5.66 (m, 0.4 H), 5.47-5.39 (m, 0.6 H), 5.15-4.97 (m, 2 H), 4.74 (d, J = 14.5 Hz, 0.6 H), 4.56-4.53 (m, 1.4 H), $4.49(\mathrm{~s}, 0.5 \mathrm{H}), 4.32$ (d, J = 14.5 Hz, 0.6 H), 4.22-4.17 (m, $0.7 \mathrm{H}), 4.03$ (d, J $=17 \mathrm{~Hz}, 0.5 \mathrm{H}), 3.65(\mathrm{dd}, J=6.5,15 \mathrm{~Hz}, 0.5 \mathrm{H}), 3.53(\mathrm{~d}, J=5.5 \mathrm{~Hz}, 1.2 \mathrm{H}), 3.13-3.02(\mathrm{~m}, 2 \mathrm{H}) ;{ }^{13} \mathrm{C}$ NMR (125 MHz, $\left.\mathrm{CDCl}_{3}\right) \delta 172.9,172.8,146.6,146.5,137.4,137.3,136.9,136.2,132.5,132.4$, $129.54,129.50,129.46,129.43,129.0,128.7,128.60,128.56,128.4,127.7,127.5,126.9,126.8,126.5$, 118.4, 118.1, 117.6, 114.3, 55.9, 55.6, 49.5, 48.7, 48.6, 46.4, 39.53, 39.49; IR (film) 3326, 3027, 1639 $\mathrm{cm}^{-1}$. Anal. Calcd for $\mathrm{C}_{25} \mathrm{H}_{26} \mathrm{~N}_{2} \mathrm{O}$ : C, 81.05; H, 7.07; N, 7.56. Found: C, 80.90; H, 7.05; N, 7.50. 


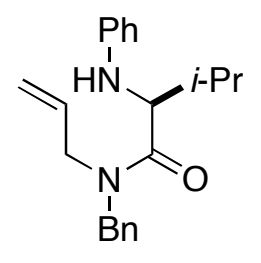

$4 \mathbf{b}$

(S)-N-Allyl- $N$-benzyl-3-methyl-2-phenylaminobutyramide (4b). General procedure 1 was employed for the coupling of (S)-3-methyl-2-phenylaminobutyric $\operatorname{acid}^{1}(1.27 \mathrm{~g}, 6.57 \mathrm{mmol})$ with $N$ benzylallylamine $(967 \mathrm{mg}, 6.57 \mathrm{mmol})$. This procedure afforded $1.37 \mathrm{~g}(65 \%)$ of the title compound as a tan solid, m.p. $48-55^{\circ} \mathrm{C}$. The enantiopurity was judged to be $99 \%$ ee by chiral hplc analysis (chiralcel OJ-H column, 10\% isopropanol/hexanes, $1 \mathrm{~mL} / \mathrm{min}$, RT = $7.40 \mathrm{~min}$ and $10.00 \mathrm{~min}$ ). This molecule was observed as a 1.5:1 mixture of rotamers; data are for the mixture. ${ }^{1} \mathrm{H} \mathrm{NMR}(500 \mathrm{MHz}$, $\left.\mathrm{CDCl}_{3}\right) \delta 7.29-7.24(\mathrm{~m}, 3 \mathrm{H}), 7.16-7.14(\mathrm{~m}, 2 \mathrm{H}), 7.09-7.06(\mathrm{~m}, 2 \mathrm{H}), 6.73-6.66$ (m, $\left.2 \mathrm{H}\right), 6.54$ (d, 1 H), 5.76-5.66 (m, 1 H), 5.21-5.04 (m, 2 H), $4.88(\mathrm{~d}, J=14.5 \mathrm{~Hz}, 0.7 \mathrm{H}), 4.70(\mathrm{~d}, J=17 \mathrm{~Hz}, 0.4 \mathrm{H})$, 4.46-4.28 (m, $2 \mathrm{H}), 4.15$ (s, $1 \mathrm{H}), 3.97-3.93(\mathrm{dd}, J=3.0,17 \mathrm{~Hz}, 0.7 \mathrm{H}), 3.84-3.79$ (dd, $J=3.0,17 \mathrm{~Hz}$, $0.7 \mathrm{H}), 3.60(\mathrm{dd}, J=6.5,15 \mathrm{~Hz}, 0.5 \mathrm{H}), 2.09-2.08(\mathrm{~m}, 1 \mathrm{H}), 1.06-1.00(\mathrm{~m}, 6 \mathrm{H}) ;{ }^{13} \mathrm{C} \mathrm{NMR}(125 \mathrm{MHz}$, $\left.\mathrm{CDCl}_{3}\right) \delta 173.6,173.5,148.25,148.21,137.2,136.2,132.7,132.6,129.2,128.9,128.6,128.1,127.7$, 127.4, 126.6, 118.09, 118,07, 117.9, 117.7, 114.5, 114.4, 59.5, 59.2, 49.9, 48.8, 48.1, 48.0, 32.3, 32.2, 20.2, 20.1, 17.72, 17.68; IR (film) 3350, 2962, $1638 \mathrm{~cm}^{-1}$. Anal. Calcd for $\mathrm{C}_{21} \mathrm{H}_{26} \mathrm{~N}_{2} \mathrm{O}: \mathrm{C}, 78.22 ; \mathrm{H}$, $8.13 ;$ N, 8.69. Found: C, 77.96; H, 8.12; N, 8.68.

General Procedure 2: Reduction of $N$-Allyl- $N$-Benzyl- $N$ '-Phenyl Amino Amides (4) to $N$-Allyl- $N$ Benzyl- $N$ '-Phenyl-1,2-Diamines (1). A flame-dried flask equipped with a magnetic stirbar was cooled under a stream of nitrogen and charged with the $N$-allyl- $N$-benzyl- $N$ '-phenyl amino amide substrate (1.0 equiv). Diethyl ether was added to provide a $0.5 \mathrm{M}$ solution, which was cooled to $0{ }^{\circ} \mathrm{C}$ with stirring. A solution of lithium aluminum hydride in diethyl ether ( $1 \mathrm{M}, 2$ equiv) was added dropwise, and the resulting mixture was stirred at $0{ }^{\circ} \mathrm{C}$ until the starting amide was completely consumed as judged by TLC analysis (ca. $2 \mathrm{~h}$ ). Water $(2 \mathrm{~mL})$ was added dropwise, followed by $10 \mathrm{M}$ aqueous $\mathrm{NaOH}(5 \mathrm{~mL})$, and additional water $(2 \mathrm{~mL})$. The resulting suspension was stirred at $\mathrm{rt}$ for 5-10 minutes, then decanted, dried over anhydrous sodium sulfate, filtered, and concentrated in vacuo. The crude product was purified by flash chromatography on silica gel. 


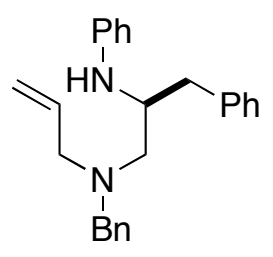

$1 \mathbf{a}$

$(S)-N^{1}$-Allyl- $N^{1}$-benzyl-3, $N^{2}$-diphenyl-propane-1,2-diamine (1a). General procedure 2 was conducted using (S)- $N$-allyl- $N$-benzyl-3-phenyl-2-phenylaminopropionamide $4 \mathbf{a}(3.79 \mathrm{~g}, 10.22 \mathrm{mmol})$ as substrate. This procedure afforded $3.13 \mathrm{~g}(86 \%)$ of the title compound as a yellow oil that was judged to be $98 \%$ ee by chiral hplc analysis (chiralcel OD-H, $0.5 \%$ isopropanol/hexanes, $1 \mathrm{~mL} / \mathrm{min}$, RT = $11.15 \mathrm{~min}$ and $12.72 \mathrm{~min}),[\alpha]_{\mathrm{D}}^{23}-43.08^{\circ}\left(c 0.26, \mathrm{CH}_{2} \mathrm{Cl}_{2}\right) .{ }^{1} \mathrm{H} \mathrm{NMR}\left(400 \mathrm{MHz}, \mathrm{CDCl}_{3}\right) \delta 7.31-7.23$ $(\mathrm{m}, 8 \mathrm{H}), 7.21-7.14(\mathrm{~m}, 5 \mathrm{H}), 6.68(\mathrm{t}, J=7.6 \mathrm{~Hz}, 1 \mathrm{H}), 6.60(\mathrm{~d}, J=8.4 \mathrm{~Hz}, 1 \mathrm{H}), 5.89-5.79(\mathrm{~m}, 1 \mathrm{H})$, 5.14-5.11 (m, $2 \mathrm{H}), 3.93(\mathrm{~s}, 1 \mathrm{H}), 3.72-3.65(\mathrm{~m}, 1 \mathrm{H}), 3.62(\mathrm{~d}, J=13.6 \mathrm{~Hz}, 1 \mathrm{H}), 3.51(\mathrm{~d}, J=13.6 \mathrm{~Hz}$, $1 \mathrm{H}), 3.14-3.01(\mathrm{~m}, 2 \mathrm{H}), 2.92(\mathrm{dd}, J=5.2,14.0 \mathrm{~Hz}, 1 \mathrm{H}), 2.80(\mathrm{dd}, J=7.2,14.4 \mathrm{~Hz}, 1 \mathrm{H}), 2.57-2.47$ $(\mathrm{m}, 2 \mathrm{H}) ;{ }^{13} \mathrm{C} \mathrm{NMR}\left(100 \mathrm{MHz}, \mathrm{CDCl}_{3}\right) \delta 148.1,139.3,138.7,135.5,129.6,129.4,129.2,128.4,127.2$, $126.3,118.0,117.3,113.4,58.7,57.4,56.9,52.3,39.2$ (one aromatic carbon signal is absent due to incidental equivalence); IR (film) 3400, 2957, $1600 \mathrm{~cm}^{-1}$. Anal. Calcd for $\mathrm{C}_{25} \mathrm{H}_{28} \mathrm{~N}_{2}$ : C, 84.23; H, 7.92; N, 7.86. Found: C, 84.23; H, 7.98; N, 7.87.

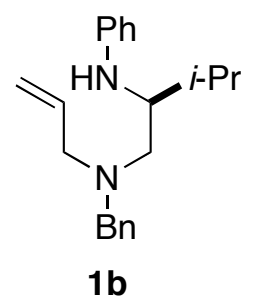

$(S)-N^{1}$-Allyl- $N^{1}$-benzyl-3-methyl- $N^{2}$-phenyl-butane-1,2-diamine (1b) General procedure 2 was conducted using $(S)-N$-allyl- $N$-benzyl-3-methyl-2-phenylaminobutyramide $4 \mathbf{b}$ (1.34 g, $4.16 \mathrm{mmol})$ as substrate. This procedure afforded $1.13 \mathrm{~g}(88 \%)$ of the title compound as a yellow oil that was judged to be $99 \%$ ee by chiral hplc analysis (chiralcel OD-H, $0.1 \%$ isopropanol $/$ hexanes, $0.2 \mathrm{~mL} / \mathrm{min}, \mathrm{RT}=$ $31.65 \mathrm{~min}$ and $35.18 \mathrm{~min}),[\alpha]_{\mathrm{D}}^{23}-59.5^{\circ}\left(c 0.98, \mathrm{CH}_{2} \mathrm{Cl}_{2}\right) .{ }^{1} \mathrm{H} \mathrm{NMR}\left(500 \mathrm{MHz}, \mathrm{CDCl}_{3}\right) \delta 7.31-7.23$ $(\mathrm{m}, 5 \mathrm{H}), 7.22-7.12(\mathrm{~m}, 2 \mathrm{H}), 6.65(\mathrm{t}, J=7.5,1 \mathrm{H}), 6.56(\mathrm{~d}, J=8.5 \mathrm{~Hz}, 2 \mathrm{H}), 5.88(\mathrm{ddt}, J=6.0,10.5$, $16.5 \mathrm{~Hz}, 1 \mathrm{H}), 5.18-5.13(\mathrm{~m}, 2 \mathrm{H}), 3.72(\mathrm{~s}, 1 \mathrm{H}), 3.63(\mathrm{~d}, J=13.5 \mathrm{~Hz}, 1 \mathrm{H}), 3.50(\mathrm{~d}, J=13.5 \mathrm{~Hz}, 1 \mathrm{H})$, 3.37-3.33 (m, $1 \mathrm{H}), 3.11(\mathrm{dd}, J=6.0,14.5 \mathrm{~Hz}, 1 \mathrm{H}), 3.04(\mathrm{dd}, J=7.0,13.5 \mathrm{~Hz}, 1 \mathrm{H}), 2.52-2.44(\mathrm{~m}, 2$ $\mathrm{H}), 2.13-2.06(\mathrm{~m}, 1 \mathrm{H}), 0.87(\mathrm{dd}, J=4.5,7.0 \mathrm{~Hz}, 6 \mathrm{H}) ;{ }^{13} \mathrm{C} \mathrm{NMR}\left(100 \mathrm{MHz}, \mathrm{CDCl}_{3}\right) \delta 149.1,139.7$, 136.0, 129.4, 129.2, 128.4, 127.1, 117.8, 116.8, 113.2, 58.9, 57.5, 55.9, 54.3, 29.5, 18.6, 17.5; IR (film) 
3400, 3025, $1601 \mathrm{~cm}^{-1}$. Anal. Calcd for $\mathrm{C}_{21} \mathrm{H}_{28} \mathrm{~N}_{2}$ : C, 81.77; H, 9.15; N, 9.08. Found: C, 81.73; H, $9.21 ; \mathrm{N}, 9.09$.

General Procedure 3: Conversion of $N$-Boc Amino Acids (5) to $N$-Boc- $N$ '-Allyl-1,2-Diamines (7). A flame-dried flask equipped with a magnetic stirbar was cooled under a stream of nitrogen and charged with the $N$-boc amino acid (1.0 equiv), DCC (2.0 equiv) and a sufficient volume of dichloromethane to provide a solution with a $0.5 \mathrm{M}$ amine concentration. The solution was stirred for $30 \mathrm{~min}$ at $\mathrm{rt}$ then the appropriate allylamine derivative (1.0 equiv) was added. The resulting mixture was stirred at room temperature for $12 \mathrm{~h}$ then filtered to remove the dicyclohexylurea byproduct. The resulting solution was washed with brine, dried over anhydrous sodium sulfate, and concentrated in vасио. The crude $N$-boc- $N$ '-allyl amino amide product was purified by flash chromatography on silica gel. In some cases the purified product was contaminated with small amounts of dicyclohexyl urea. This material was carried on without further purification.

A flame-dried flask equipped with a magnetic stirbar was cooled under a stream of nitrogen and charged with the $N$-boc- $N$ '-allyl amino amide (1.0 equiv). Diethyl ether was added to provide a $0.5 \mathrm{M}$ solution, which was cooled to $0{ }^{\circ} \mathrm{C}$. A solution of lithium aluminum hydride in diethyl ether (1 M, 2.0 equiv) was added dropwise and the reaction was stirred at $0{ }^{\circ} \mathrm{C}$ until the starting amide was consumed as judged by TLC analysis (ca. $3 \mathrm{~h}$ ). Water $(2 \mathrm{~mL}$ ) was added dropwise, followed by $10 \mathrm{M}$ aqueous $\mathrm{NaOH}(5 \mathrm{~mL})$, and additional water $(2 \mathrm{~mL})$. The resulting suspension was stirred at $\mathrm{rt}$ for 5-10 minutes, then decanted, dried over anhydrous sodium sulfate, filtered, and concentrated in vacuo. The crude product was purified by flash chromatography on silica gel.

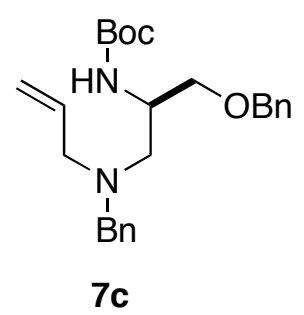

(R)-tert-Butyl 1-[allyl(benzyl)amino]-3-(benzyloxy)propan-2-ylcarbamate (7c). General procedure 3 was used for the coupling of (S)-3-(benzyloxy)-2-(tert-butoxycarbonylamino)propanoic acid (5.99 g, $20.28 \mathrm{mmol})$ with $N$-benzylallylamine $(2.98 \mathrm{~g}, 20.28 \mathrm{mmol})$. This procedure afforded $7.67 \mathrm{~g}(89 \%)$ of (S)-tert-butyl 1-[allyl(benzyl)amino]-3-(benzyloxy)-1-oxopropan-2-ylcarbamate as a white solid, which was contaminated with ca. $15 \%$ dicyclohexylurea. This material was carried on without further purification. The enantiopurity was judged to be $98 \%$ ee by chiral hplc analysis (chiralcel OD column, 
$5 \%$ isopropanol/hexanes, $1 \mathrm{~mL} / \mathrm{min}, \mathrm{RT}=7.19 \mathrm{~min}$ and $9.13 \mathrm{~min}$ ). This molecule was isolated as a 4.6:1 mixture of rotamers; data are for the mixture. ${ }^{1} \mathrm{H} \mathrm{NMR}\left(400 \mathrm{MHz}, \mathrm{CDCl}_{3}\right) \delta 7.38-7.28(\mathrm{~m}, 6 \mathrm{H})$, 7.20-7.17 (m, 4 H), 5.82-5.69 (m, $1 \mathrm{H}), 5.43(\mathrm{~m}, 0.8 \mathrm{H})$, 5.26-5.07 (m, $2 \mathrm{H})$, 4.99-4.97 (m, 0.2 H), 4.71-4.53 (m, 3.8 H), 4.44-4.40 (m, 0.2 H), 4.17-4.07 (m, 0.2 H), 4.02-3.96 (m, 1.8 H), 3.86-3.79 (m, 2.8 H), 3.71-3.66 (m, $0.2 \mathrm{H}), 1.43(\mathrm{~m}, 9 \mathrm{H})$.

The (S)-tert-butyl 1-[allyl(benzyl)amino]-3-(benzyloxy)-1-oxopropan-2-ylcarbamate product of the DCC coupling reaction $(7.65 \mathrm{~g}, 18.0 \mathrm{mmol})$ was reduced following general procedure 3 . This procedure afforded $4.83 \mathrm{~g}(65 \%)$ of the title compound as a colorless oil. ${ }^{1} \mathrm{H} \mathrm{NMR}\left(400 \mathrm{MHz}, \mathrm{CDCl}_{3}\right) \delta$ 7.31-7.17 (m, $10 \mathrm{H}), 5.80(\mathrm{dt}, J=6.8,10.4 \mathrm{~Hz}, 1 \mathrm{H}), 5.15-5.08$ (m, $2 \mathrm{H}), 4.81$ (s, $1 \mathrm{H}), 4.42-4.35$ (m, $2 \mathrm{H}), 3.80(\mathrm{~s}, 1 \mathrm{H}), 3.65-3.58(\mathrm{~m}, 2 \mathrm{H}), 3.51-3.44(\mathrm{~m}, 2 \mathrm{H}), 3.11(\mathrm{dd}, J=8.0,14.4 \mathrm{~Hz}, 1 \mathrm{H}), 3.01$ (dd, $J=6.4,14.4 \mathrm{~Hz}, 1 \mathrm{H}), 2.64(\mathrm{dd}, J=7.6,12.8 \mathrm{~Hz}, 1 \mathrm{H}), 2.47(\mathrm{dd}, J=6.0,12.4 \mathrm{~Hz}, 1 \mathrm{H}), 1.41(\mathrm{~s}, 9 \mathrm{H})$;

${ }^{13} \mathrm{C}$ NMR $\left(100 \mathrm{MHz}, \mathrm{CDCl}_{3}\right) \delta 155.9,139.7,138.5,136.0,129.1,128.5,128.4,127.80,127.77,127.1$, 117.7, 79.3, 73.4, 70.2, 58.6, 57.3, 54.5, 48.9, 28.6; IR (film) 3436, 2976, $1713 \mathrm{~cm}^{-1}$; MS (ESI) 411.2637 (411.2648 calcd for $\mathrm{C}_{25} \mathrm{H}_{34} \mathrm{~N}_{2} \mathrm{O}_{3}, \mathrm{M}+\mathrm{H}^{+}$).

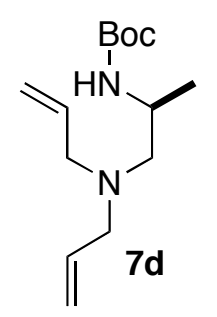

(S)-tert-Butyl 1-(diallylamino)propan-2-ylcarbamate (7d). General procedure 3 was used for the coupling of (S)-2-tert-butoxycarbonylaminopropionic acid (1.16 g, $6.13 \mathrm{mmol})$, with diallylamine (595 $\mathrm{mg}, 754 \mu \mathrm{L}, 6.13 \mathrm{mmol})$. This procedure afforded $1.64 \mathrm{~g}$ (100\%) of (S)-tert-butyl 1-(diallylamino)-1oxopropan-2-ylcarbamate as a white solid, which was contaminated with $5 \%$ dicyclohexylurea. This material was carried on without further purification. This molecule was observed as a 5:1 mixture of rotamers. Data are for the mixture ${ }^{1} \mathrm{H}$ NMR $\left(500 \mathrm{MHz}, \mathrm{CDCl}_{3}\right) \delta 5.83-5.70(\mathrm{~m}, 2 \mathrm{H}), 5.39(\mathrm{~d}, J=7.5$ Hz, 0.8 H), 5.25-5.11 (m, 4 H), 4.99-4.96 (m, 0.2 H), 4.59-4.57 (m, 0.8 H), 4.43-4.41 (m, 0.2 H), 4.18-4.10 (m, 0.2 H), 4.06-3.88 (m, 3.6 H), 3.70-3.60 (m, 0.2 H), $1.43(\mathrm{~s}, 9 \mathrm{H}), 1.31(\mathrm{~d}, J=6.5 \mathrm{~Hz}, 3$ $\mathrm{H})$.

The (S)-tert-butyl 1-(diallylamino)-1-oxopropan-2-ylcarbamate product of the DCC coupling reaction $(1.64 \mathrm{~g}, 6.16 \mathrm{mmol})$ was reduced with lithium aluminium hydride following general procedure 3. This procedure afforded $1.13 \mathrm{~g}(72 \%)$ of the title compound as a white solid, m.p. $45-47{ }^{\circ} \mathrm{C} .{ }^{1} \mathrm{H}$ 
NMR (500 MHz, $\left.\mathrm{CDCl}_{3}\right) \delta 5.85-5.77(\mathrm{~m}, 2 \mathrm{H}), 5.18-5.11(\mathrm{~m}, 4 \mathrm{H}), 4.68(\mathrm{~s}, 1 \mathrm{H}), 3.67-3.63(\mathrm{~m}, 1 \mathrm{H})$, 3.12 (dd, $J=6.0,14 \mathrm{~Hz}, 2 \mathrm{H}), 3.04$ (dd, $J=6.5,14 \mathrm{~Hz}, 2 \mathrm{H}), 2.40-2.30$ (m, $2 \mathrm{H}), 1.44$ (s, $9 \mathrm{H}), 1.12$ (d, $J=6.5 \mathrm{~Hz}, 3 \mathrm{H}) ;{ }^{13} \mathrm{C} \mathrm{NMR}\left(100 \mathrm{MHz}, \mathrm{CDCl}_{3}\right) \delta 156.0,135.9,117.7,79.1,58.7,57.3,44.8,28.7,19.7$; IR (film) 3326, 2930, $1690 \mathrm{~cm}^{-1}$; MS (EI) 254.2003 (254.1994 calcd for $\mathrm{C}_{14} \mathrm{H}_{26} \mathrm{~N}_{2} \mathrm{O}_{2}, \mathrm{M}+\mathrm{H}^{+}$).

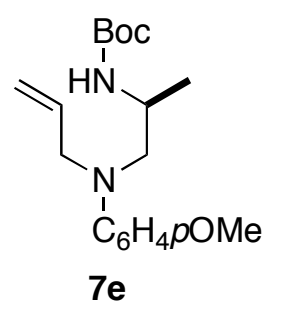

(S)-tert-Butyl 1-[allyl(4-methoxyphenyl)amino]propan-2-ylcarbamate (7e). General procedure 3 was used for the coupling of (S)-2-tert-butoxycarbonylaminopropionic acid (828 $\mathrm{mg}, 4.38 \mathrm{mmol})$ and $N$-p-methoxyphenylallylamine ${ }^{1,3}(710 \mathrm{mg}, 4.38 \mathrm{mmol})$. This procedure afforded $1.28 \mathrm{~g}(88 \%)$ of $(S)$ tert-butyl 1-[allyl(4-methoxyphenyl)amino]-1-oxopropan-2-ylcarbamate as a white solid, which was contaminated with ca. $15 \%$ of dicyclohexylurea. ${ }^{1} \mathrm{H}$ NMR $\left(500 \mathrm{MHz}, \mathrm{CDCl}_{3}\right) \delta 7.13(\mathrm{~d}, J=8.5 \mathrm{~Hz}, 2$ H), $6.92(\mathrm{~d}, J=9.5 \mathrm{~Hz}, 2 \mathrm{H}), 5.82(\mathrm{ddt}, J=6.0,11.0,17 \mathrm{~Hz}, 1 \mathrm{H}), 5.28(\mathrm{~d}, J=8.0 \mathrm{~Hz}, 1 \mathrm{H}), 5.12(\mathrm{dd}, J$ $=1.5,11 \mathrm{~Hz}, 1 \mathrm{H}), 5.07(\mathrm{dd}, J=1.5,17 \mathrm{~Hz}, 1 \mathrm{H}), 4.34-4.27(\mathrm{~m}, 2 \mathrm{H}), 4.18-4.11(\mathrm{~m}, 1 \mathrm{H}), 3.82(\mathrm{~s}, 3$ H), $1.41(\mathrm{~s}, 9 \mathrm{H}), 1.11(\mathrm{~d}, J=6.5 \mathrm{~Hz}, 3 \mathrm{H})$.

The (S)-tert-butyl 1-[allyl(4-methoxyphenyl)amino-1-oxopropan-2-ylcarbamate coupling product of the DCC reaction $(1.28 \mathrm{~g}, 3.83 \mathrm{mmol})$ was reduced with lithium aluminum hydride following general procedure 3. This procedure afforded $4.83 \mathrm{~g}(65 \%)$ of the title compound as a white solid, m.p.

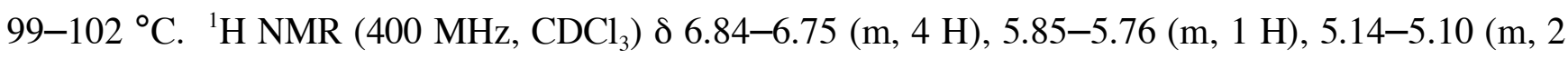
H), 4.46 (s, br, $1 \mathrm{H}), 3.95-3.76(\mathrm{~m}, 3 \mathrm{H}), 3.74(\mathrm{~s}, 3 \mathrm{H}), 3.36(\mathrm{dd}, J=6.0,14.4 \mathrm{~Hz}, 1 \mathrm{H}), 3.05(\mathrm{dd}, J=$ 5.6, $14.4 \mathrm{~Hz}, 1 \mathrm{H}), 1.43$ (s, $9 \mathrm{H}), 1.16$ (d, $J=6.8 \mathrm{~Hz}, 3 \mathrm{H}) ;{ }^{13} \mathrm{C}$ NMR $\left(100 \mathrm{MHz}, \mathrm{CDCl}_{3}\right) \delta$ 155.6, 152.0, 143.5, 134.5, 116.7, 115.2, 114.9, 57.3, 55.9, 55.3, 45.7, 29.9, 28.6, 19.2; IR (film) 3357, $1676 \mathrm{~cm}^{-1}$; MS (EI) 320.2095 (320.2100 calcd for $\mathrm{C}_{18} \mathrm{H}_{28} \mathrm{~N}_{2} \mathrm{O}_{3}$ ).

General Procedure 4: Conversion of $N$-Boc- $N$ '-Allyl-1,2-diamines (7) to $N$-aryl- $N$ '-Allyl-1,2diamines (1). A flask equipped with magnetic stirbar was charged with the appropriate $N$-boc- $N$ '-allyl1,2-diamine (1.0 equiv) and a sufficient volume of dioxane to provide a $0.1 \mathrm{M}$ solution. A solution of 4 $\mathrm{M}$ aqueous $\mathrm{HCl}$ (33 equiv) was added and the reaction mixture was heated to $50{ }^{\circ} \mathrm{C}$ for $2 \mathrm{~h}$. The reaction mixture was cooled to room temperature and $\mathrm{NH}_{4} \mathrm{OH}$ was added dropwise until the solution 
$\mathrm{pH}$ was $>11$. The resulting mixture was extracted with ethyl acetate, and the combined organic layers were washed with brine, dried over anhydrous sodium sulfate, and concentrated in vacuo to afford the corresponding primary amine product. The crude product was immediately carried on without further purification.

A flame dried Schlenk tube equipped with a magnetic stirbar was charged with $\operatorname{Pd}_{2}(\mathrm{dba})_{3}(1 \mathrm{~mol} \%$ complex, $2 \mathrm{~mol} \% \mathrm{Pd}),( \pm)$-BINAP (2 mol \%), sodium tert-butoxide (1.2 equiv), the appropriate aryl bromide (1.0 equiv), and a $0.5 \mathrm{M}$ solution of the primary amine (1.0 equiv) in toluene. The reaction mixture was heated to $80{ }^{\circ} \mathrm{C}$ with stirring until the starting material had been consumed as judged by TLC analysis (ca. $6 \mathrm{~h}$ ). The mixture was cooled to $\mathrm{rt}$ and a solution of aqueous ammonium chloride was added $(4 \mathrm{~mL})$. The resulting mixture was extracted with ethyl acetate and the combined organic layers were dried over anhydrous sodium sulfate, filtered, and concentrated in vacuo. The crude product was purified by flash chromatography on silica gel.

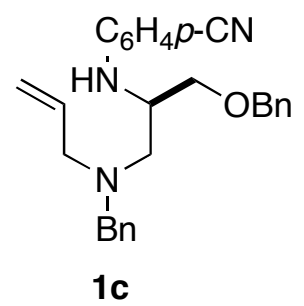

(R)-4-\{1-[Allyl(benzyl)amino]-3-(benzyloxy)propan-2-ylamino\}benzonitrile (1c). General procedure 4 was used for the deprotection of $(R)$-tert-butyl 1-[allyl(benzyl)amino]-3(benzyloxy)propan-2-ylcarbamate 7c $(3.9 \mathrm{~g}, 9.51 \mathrm{mmol})$. This procedure afforded $3.1 \mathrm{~g}(100 \%)$ of $(S)$ $N^{1}$-allyl- $N^{1}$-benzyl-3-benzyloxypropane-1,2-diamine as a yellow oil. The crude product was immediately subjected to the subsequent reaction without further purification. ${ }^{1} \mathrm{H} \mathrm{NMR}(400 \mathrm{MHz}$, $\left.\mathrm{CDCl}_{3}\right) \delta 7.37-7.21(\mathrm{~m}, 10 \mathrm{H}), 5.86(\mathrm{dt}, J=6,11 \mathrm{~Hz}, 1 \mathrm{H}), 5.17-5.12(\mathrm{~m}, 2 \mathrm{H}), 4.50(\mathrm{~s}, 2 \mathrm{H}), 3.67(\mathrm{~d}$, $J=13.5 \mathrm{~Hz}, 1 \mathrm{H}), 3.51-3.48(\mathrm{~m}, 2 \mathrm{H}), 3.30-3.26(\mathrm{~m}, 1 \mathrm{H}), 3.20-3.12(\mathrm{~m}, 2 \mathrm{H}), 3.00(\mathrm{dd}, J=5.4,7.5$ $\mathrm{Hz}, 1 \mathrm{H}), 2.46-2.38(\mathrm{~m}, 2 \mathrm{H}), 1.60$ (s, $2 \mathrm{H})$; MS (ESI) 311.2116 (311.2123 calcd for $\mathrm{C}_{20} \mathrm{H}_{26} \mathrm{~N}_{2} \mathrm{O}, \mathrm{M}+$ $\mathrm{H}^{+}$.

General procedure 4 was used for the $N$-arylation of $(S)$ - $N^{1}$-allyl- $N^{1}$-benzyl-3-benzyloxypropane1,2-diamine (900 mg, $2.89 \mathrm{mmol}$ ) with 4-bromobenzonitrile (527 mg, $2.89 \mathrm{mmol})$. This procedure afforded $864 \mathrm{mg}(73 \%)$ of the title compound as an orange oil. The enantiopurity was judged to be 98\% ee by chiral hplc analysis (chiralcel AD column, $0.7 \%$ isopropanol/hexanes, $1 \mathrm{~mL} / \mathrm{min}$, RT = $24.75 \mathrm{~min}$ and $28.10 \mathrm{~min}),[\alpha]_{\mathrm{D}}^{23}-25.59^{\circ}\left(c 0.79, \mathrm{CH}_{2} \mathrm{Cl}_{2}\right)$. ${ }^{1} \mathrm{H} \mathrm{NMR}\left(400 \mathrm{MHz}, \mathrm{CDCl}_{3}\right) \delta 7.35-7.28$ 
$(\mathrm{m}, 12 \mathrm{H}), 6.40(\mathrm{~d}, J=8.5 \mathrm{~Hz}, 2 \mathrm{H}), 5.85(\mathrm{dt}, J=6.5,10.5 \mathrm{~Hz}, 1 \mathrm{H}), 5.22-5.16(\mathrm{~m}, 2 \mathrm{H}), 4.54(\mathrm{~d}, J=$ $6.5 \mathrm{~Hz}, 1 \mathrm{H}), 4.49-4.44$ (m, $2 \mathrm{H}), 3.67$ (dd, $J=3.0,9.0 \mathrm{~Hz}, 1 \mathrm{H}), 3.65-3.56$ (m, $2 \mathrm{H}), 3.53-3.46$ (m, 2 H), 3.17-3.10 (m, $2 \mathrm{H}), 2.74(\mathrm{dd}, J=7.5,13.5 \mathrm{~Hz}, 1 \mathrm{H}), 2.60(\mathrm{dd}, J=6.5,13.5 \mathrm{~Hz}, 1 \mathrm{H}) ;{ }^{13} \mathrm{C}$ NMR $\left(100 \mathrm{MHz}, \mathrm{CDCl}_{3}\right) \delta 151.0,139.5,138.0,135.6,133.8,129.1,128.6,128.5,127.99,127.90,127.4$, 120.7, 118.1, 112.7, 98.6, 73.5, 69.9, 59.3, 58.3, 54.3, 51.4; IR (film) 3365, 2211, $1606 \mathrm{~cm}^{-1}$; MS (ESI) 412.2383 (412.2389 calcd for $\mathrm{C}_{27} \mathrm{H}_{29} \mathrm{~N}_{3} \mathrm{O}, \mathrm{M}+\mathrm{H}^{+}$).

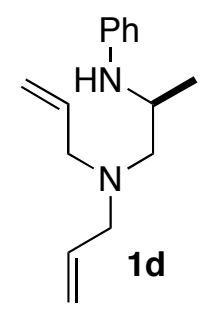

(S)- $N^{1}, N^{1}$-Diallyl- $N^{2}$-phenylpropane-1,2-diamine (1d). General procedure 4 was used for the deprotection of $1.08 \mathrm{~g}$ ( $4.24 \mathrm{mmol})$ of $(S)$-tert-butyl 1-(diallylamino)propan-2-ylcarbamate 7d (1.08 g, $4.24 \mathrm{mmol})$. This procedure afforded $654 \mathrm{mg}(85 \%)$ of $(S)-N^{1}, N^{1}$-diallylpropane-1,2-diamine as a yellow oil. The crude product was immediately subjected to the subsequent reaction without further purification. ${ }^{1} \mathrm{H}$ NMR (500 MHz, $\mathrm{CDCl}_{3}$ ) $\delta$ 5.95-5.87 (m, $\left.2 \mathrm{H}\right), 5.19-5.15(\mathrm{~m}, 4 \mathrm{H}), 3.70$ (s, br, $\left.2 \mathrm{H}\right)$, 3.36-3.30 (m, $1 \mathrm{H}), 3.24-3.20$ (m, 2 H), 3.13-3.10 (m, 2 H), 2.66 (dd, $J=10.5,15 \mathrm{~Hz}, 1 \mathrm{H}), 2.53$ (dd, $J=4.5,14 \mathrm{~Hz}, 1 \mathrm{H}) .1 .40(\mathrm{~d}, J=6.5 \mathrm{~Hz}, 3 \mathrm{H})$.

General procedure 4 was used for the $N$-arylation of $(S)-N^{1}, N^{1}$-diallylpropane-1,2-diamine (379 $\mathrm{mg}, 2.46 \mathrm{mmol})$ with bromobenzene ( $386 \mathrm{mg}, 2.46 \mathrm{mmol})$. This procedure afforded $390 \mathrm{mg}(69 \%)$ of the title compound as a yellow oil. The enantiopurity was judged to be $99 \%$ ee by chiral hplc analysis (chiralcel OJ-H column, $1 \% \mathrm{IPA} / \mathrm{hexanes,} 0.2 \mathrm{~mL} / \mathrm{min}, \mathrm{RT}=38.71 \mathrm{~min}$ and $43.68 \mathrm{~min}$ ), $[\alpha]^{23}{ }_{\mathrm{D}}-4.61^{\circ}$ (c 0.23, $\left.\mathrm{CH}_{2} \mathrm{Cl}_{2}\right) .{ }^{1} \mathrm{H}$ NMR $\left(500 \mathrm{MHz}, \mathrm{CDCl}_{3}\right) \delta 7.18-7.15(\mathrm{~m}, 2 \mathrm{H}), 6.69(\mathrm{t}, J=7.5 \mathrm{~Hz}, 1 \mathrm{H}), 6.64(\mathrm{~d}$, $J=8.5 \mathrm{~Hz}, 2 \mathrm{H}), 5.83$ (ddt, $J=6.5,7.0,10 \mathrm{~Hz}, 2 \mathrm{H}), 5.18-5.12(\mathrm{~m}, 4 \mathrm{H}), 4.20(\mathrm{~s}, 1 \mathrm{H}), 3.50-3.43$ (m, $1 \mathrm{H}), 3.17(\mathrm{dd}, J=6.0,14 \mathrm{~Hz}, 2 \mathrm{H}), 3.04(\mathrm{dd}, J=7.0,14.5 \mathrm{~Hz}, 2 \mathrm{H}), 2.54(\mathrm{dd}, J=8.5,13 \mathrm{~Hz}, 1 \mathrm{H})$, $2.42(\mathrm{dd}, J=6.0,13 \mathrm{~Hz}, 1 \mathrm{H}), 1.19(\mathrm{~d}, J=6.0 \mathrm{~Hz}, 3 \mathrm{H}),{ }^{13} \mathrm{C}\left(100 \mathrm{MHz}, \mathrm{CDCl}_{3}\right) \delta 148.4,135.7,129.3$, 117.7, 117.3, 113.7, 59.0, 57.2, 46.6, 19.9; IR (film) 3350, 2924, $1602 \mathrm{~cm}^{-1}$; MS (EI) 230.1787 (230.1783 calcd for $\mathrm{C}_{15} \mathrm{H}_{22} \mathrm{~N}_{2}$ ). 


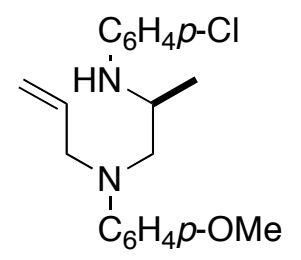

$1 \mathrm{e}$

\section{(S)- $N^{1}$-Allyl- $N^{2}$-(4-chlorophenyl)- $N^{1}$-(4-methoxyphenyl)propane-1,2-diamine}

(1e) General procedure 4 was used for the deprotection of (S)-tert-butyl 1-[allyl(4-methoxyphenyl)amino]propan-2-

ylcarbamate 7 e $(513 \mathrm{mg}, 1.60 \mathrm{mmol})$. This procedure afforded $318 \mathrm{mg}(91 \%)$ of $(S)-N^{1}$-allyl- $N^{1}$-(4methoxyphenyl)propane-1,2-diamine as a yellow oil. The crude product was immediately subjected to the subsequent reaction without further purification. ${ }^{1} \mathrm{H} \mathrm{NMR}\left(500 \mathrm{MHz}, \mathrm{CDCl}_{3}\right) \delta 6.81(\mathrm{~d}, J=9.0 \mathrm{~Hz}$, $2 \mathrm{H}), 6.75(\mathrm{~d}, J=9.0 \mathrm{~Hz}, 2 \mathrm{H}), 5.86-5.79(\mathrm{~m}, 1 \mathrm{H}), 5.14-5.11(\mathrm{~m}, 2 \mathrm{H}), 3.90(\mathrm{dd}, J=1.5,5.0 \mathrm{~Hz}, 2 \mathrm{H})$, $3.75(\mathrm{~s}, 3 \mathrm{H}), 3.26-3.21(\mathrm{~m}, 1 \mathrm{H}), 3.20(\mathrm{~d}, J=4.5 \mathrm{~Hz}, 1 \mathrm{H}), 3.00-2.96(\mathrm{~m}, 1 \mathrm{H}), 1.60$ (s, br, $2 \mathrm{H}), 1.09$ $(\mathrm{d}, J=6.0 \mathrm{~Hz}, 3 \mathrm{H})$.

General procedure 4 was used for the $N$-arylation of $(S)-N^{1}$-allyl- $N^{1}$-(4-methoxyphenyl)propane1,2-diamine (64 mg, $0.29 \mathrm{mmol})$ with 4-chlorobromobenzene $(55 \mathrm{mg}, 0.29 \mathrm{mmol})$. This procedure afforded $65 \mathrm{mg}(69 \%)$ of the title compound as a yellow oil. The enantiopurity was judged to be $98 \%$ ee by chiral hplc analysis (chiralcel OD column, $1 \%$ isopropanol/hexanes, $0.2 \mathrm{~mL} / \mathrm{min}, \mathrm{RT}=59.14 \mathrm{~min}$ and $63.37 \mathrm{~min}),[\alpha]^{23}{ }_{\mathrm{D}}+21.46^{\circ}\left(c 0.11, \mathrm{CH}_{2} \mathrm{Cl}_{2}\right) .{ }^{1} \mathrm{H}$ NMR $\left(400 \mathrm{MHz}, \mathrm{CDCl}_{3}\right) \delta 7.08(\mathrm{~d}, J=8.8 \mathrm{~Hz}, 2$ H), $6.83(\mathrm{~d}, J=9.2 \mathrm{~Hz}, 2 \mathrm{H}), 6.75(\mathrm{~d}, J=9.2 \mathrm{~Hz}, 2 \mathrm{H}), 6.48(\mathrm{~d}, J=8.8 \mathrm{~Hz}, 2 \mathrm{H}), 5.86-5.77(\mathrm{~m}, 1 \mathrm{H})$, 5.16-5.11 (m, $2 \mathrm{H}), 3.95-3.79(\mathrm{~m}, 2 \mathrm{H}), 3.77$ (s, $3 \mathrm{H}), 3.74-3.63$ (m, $2 \mathrm{H}), 3.32-3.22(\mathrm{~m}, 2 \mathrm{H}), 1.22$ $(\mathrm{d}, J=6.4 \mathrm{~Hz}, 3 \mathrm{H}) ;{ }^{13} \mathrm{C} \mathrm{NMR}\left(100 \mathrm{MHz}, \mathrm{CDCl}_{3}\right) \delta 152.5,146.4,143.6,134.6,129.2,121.9,116.9$, 116.2, 114.9, 114.6, 57.8, 55.9, 55.7, 48.0, 19.5; IR (film) 3391, 2929, $1598 \mathrm{~cm}^{-1}$; MS (EI) 330.1490 (330.1500 calcd for $\mathrm{C}_{19} \mathrm{H}_{23} \mathrm{ClN}_{2}$ ).

\section{Synthesis of Piperazines via Coupling with Aryl Bromides (Tables 1-2)}

General Procedure for Pd-Catalyzed Synthesis of Piperazines. A flame-dried Schlenk tube equipped with a magnetic stirbar was cooled under a stream of nitrogen and charged with $\mathrm{Pd}_{2}(\mathrm{dba})_{3}(1$ mol \% complex, 2 mol \% Pd), $\mathrm{P}(2 \text {-furyl })_{3}(8 \mathrm{~mol} \%)$, sodium $t$-butoxide (1.2 equiv), and the aryl bromide (1.2 equiv). The Schlenk tube was purged with nitrogen and the amine was added as a solution in toluene $\left(2.5 \mathrm{~mL} / 0.5 \mathrm{mmol}\right.$ substrate). The Schlenk tube was then heated to $105^{\circ} \mathrm{C}$ with stirring until 
the starting material has been consumed as judged by ${ }^{1} \mathrm{H}$ NMR analysis of an aliquot taken from the reaction mixture. The reaction mixture was then cooled to room temperature, saturated aqueous ammonium chloride (2-3 mL) was added, and the resulting mixture was extracted with ethyl acetate (3 x $8 \mathrm{~mL}$ ). The combined organic layers were dried over anhydrous sodium sulfate, filtered, and concentrated in vacuo. The crude product was then purified by flash chromatography on silica gel.

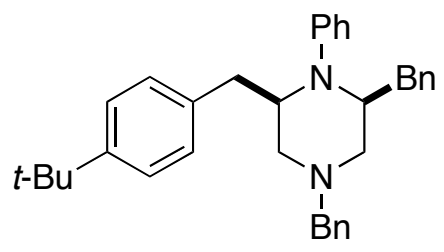

12

(2S,6R)-2,4-Dibenzyl-6-(4-tert-butylbenzyl)-1-phenylpiperazine (12). The reaction of $150 \mathrm{mg}(0.42$ $\mathrm{mmol})$ of $N^{1}$-allyl- $N^{1}$-benzyl-3, $N^{2}$-diphenylpropane-1,2-diamine (1a) with $108 \mathrm{mg}(0.51 \mathrm{mmol})$ of 4 bromo-tert-butylbenzene was conducted for $10 \mathrm{~h}$ according to the general procedure. The product was formed with $>20: 1 \mathrm{dr}$ as judged by ${ }^{1} \mathrm{H}$ NMR analysis of a sample taken from the crude reaction mixture. Upon purification, $132 \mathrm{mg}(64 \%)$ of the title compound was obtained as a white solid, m.p. $74-77{ }^{\circ} \mathrm{C}$. This material was judged to be of $>20: 1 \mathrm{dr}$ by ${ }^{1} \mathrm{H}$ NMR analysis. The enantiopurity was judged to be $99 \%$ ee by chiral hplc analysis (chiralcel OJ-H column, $2 \%$ isopropanol/hexanes, 0.1 $\mathrm{mL} / \mathrm{min}, \mathrm{RT}=55.13 \mathrm{~min}$ and $101.14 \mathrm{~min}),[\alpha]^{23}{ }_{\mathrm{D}}+5.48^{\circ}\left(c 0.27, \mathrm{CH}_{2} \mathrm{Cl}_{2}\right) .{ }^{1} \mathrm{H} \mathrm{NMR}(400 \mathrm{MHz}$, $\left.\mathrm{CDCl}_{3}\right) \delta$ 7.47-7.38 (m, $\left.7 \mathrm{H}\right), 7.24-7.17(\mathrm{~m}, 5 \mathrm{H}), 7.07-6.99(\mathrm{~m}, 6 \mathrm{H}), 6.83(\mathrm{t}, J=7.2 \mathrm{~Hz}, 1 \mathrm{H}), 3.80$ (d, $J=6.4 \mathrm{~Hz}, 2 \mathrm{H}), 3.54-3.44(\mathrm{~m}, 2 \mathrm{H}), 3.07(\mathrm{t}, J=12.8 \mathrm{~Hz}, 2 \mathrm{H}), 2.94-2.87(\mathrm{~m}, 2 \mathrm{H}), 2.79(\mathrm{t}, J=$

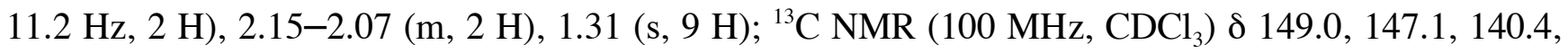
139.0, 137.3, 130.1, 129.9, 129.5, 129.1, 128.7, 128.6, 127.5, 126.2, 125.6, 117.4, 113.5, 63.2, 55.6, 55.5, 54.5, 54.1, 37.5, 36.9, 34.6, 31.6; IR (film) 2960, $1597 \mathrm{~cm}^{-1}$; MS (ESI) 489.3272 (489.3270 calcd for $\mathrm{C}_{35} \mathrm{H}_{40} \mathrm{~N}_{2}, \mathrm{M}+\mathrm{H}^{+}$). 


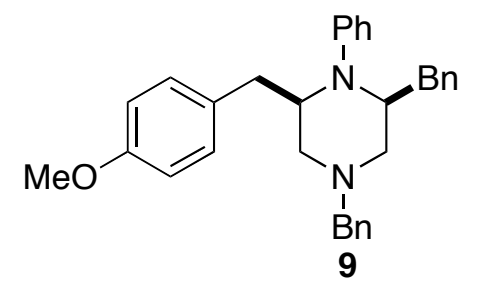

(2S,6R)-2,4-Dibenzyl-6-(4-methoxybenzyl)-1-phenylpiperazine (9). The reaction of $150 \mathrm{mg}(0.42$ mmol) of $N^{1}$-allyl- $N^{1}$-benzyl-3, $N^{2}$-diphenylpropane-1,2-diamine (1a) with $95 \mathrm{mg}(0.51 \mathrm{mmol})$ of 4 bromoanisole was conducted for $10 \mathrm{~h}$ according to the general procedure. The product was formed with $>20: 1 \mathrm{dr}$ as judged by ${ }^{1} \mathrm{H}$ NMR analysis of a sample taken from the crude reaction mixture. Upon purification, $120 \mathrm{mg}(62 \%)$ of the title compound was obtained as a white solid, m.p. $119-122^{\circ} \mathrm{C}$. This material was judged to be of $>20: 1 \mathrm{dr}$ by ${ }^{1} \mathrm{H}$ NMR analysis. The enantiopurity was judged to be $99 \%$ ee by chiral hplc analysis (chiralcel OJ-H column, $10 \%$ isopropanol/hexanes, $0.5 \mathrm{~mL} / \mathrm{min}, \mathrm{RT}=15.12$ min and $30.50 \mathrm{~min}),[\alpha]^{23}{ }_{\mathrm{D}}+13.51^{\circ}\left(c 0.48, \mathrm{CH}_{2} \mathrm{Cl}_{2}\right) .{ }^{1} \mathrm{H} \mathrm{NMR}\left(400 \mathrm{MHz}, \mathrm{CDCl}_{3}\right) \delta 7.47-7.38(\mathrm{~m}, 7$ H), 7.26-7.18 (m, $3 \mathrm{H}), 7.06(\mathrm{~d}, J=8.0 \mathrm{~Hz}, 4 \mathrm{H}), 6.95(\mathrm{~d}, J=8.4 \mathrm{~Hz}, 3 \mathrm{H}), 6.84(\mathrm{t}, J=6.0 \mathrm{~Hz}, 1 \mathrm{H})$, $6.77(\mathrm{~d}, J=8.4 \mathrm{~Hz}, 1 \mathrm{H}), 3.83-3.74(\mathrm{~m}, 5 \mathrm{H}), 3.52-3.45(\mathrm{~m}, 2 \mathrm{H}), 3.11-2.99(\mathrm{~m}, 2 \mathrm{H}), 2.89(\mathrm{~d}, J=$

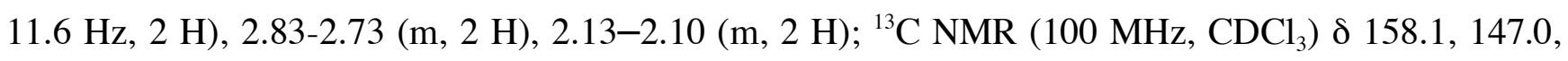
140.3, 138.9, 132.4, 130.4, 130.1, 129.9, 129.5, 128.7, 128.6, 127.4, 126.2, 117.4, 114.1, 113.5, 63.2, 55.8, 55.6, 55.4, 54.3, 54.1, 37.5, 36.5; IR (film) 3026, 2812, $1597 \mathrm{~cm}^{-1}$; MS (ESI) 463.2744 (463.2749 calcd for $\mathrm{C}_{32} \mathrm{H}_{34} \mathrm{~N}_{2} \mathrm{O}, \mathrm{M}+\mathrm{H}^{+}$).

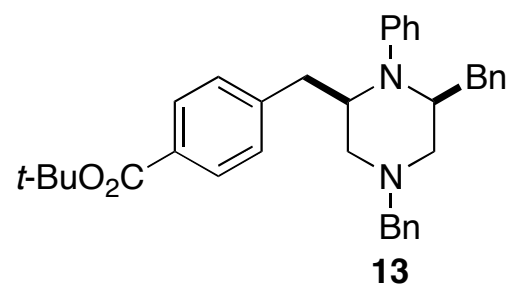

(2R,6S)-tert-butyl 4-[(4,6-dibenzyl-1-phenylpiperazin-2-yl)methyl]benzoate (13). The reaction of $150 \mathrm{mg}(0.42 \mathrm{mmol})$ of $N^{1}$-allyl- $N^{1}$-benzyl-3, $N^{2}$-diphenylpropane-1,2-diamine with $130 \mathrm{mg}(0.51$ $\mathrm{mmol}$ ) of 4-bromo-tert-butylbenzoate was conducted for $10 \mathrm{~h}$ according to the general procedure. The product was formed with $>20: 1 \mathrm{dr}$ as judged by ${ }^{1} \mathrm{H}$ NMR analysis of a sample taken from the crude reaction mixture. Upon purification, $132 \mathrm{mg}$ (59\%) of the title compound was obtained as a white solid, m.p. $75-77{ }^{\circ} \mathrm{C}$. This material was judged to be of $>20: 1 \mathrm{dr}$ by ${ }^{1} \mathrm{H}$ NMR analysis. The enantiopurity was judged to be $99 \%$ ee by chiral hplc analysis (chiralcel OD-H column, $5 \%$ isopropanol/hexanes, $2 \mathrm{~mL} / \mathrm{min}, \mathrm{RT}=1.40 \mathrm{~min}$ and $1.94 \mathrm{~min}),[\alpha]^{23}{ }_{\mathrm{D}}+35.1^{\circ}\left(c 0.30, \mathrm{CH}_{2} \mathrm{Cl}_{2}\right) .{ }^{1} \mathrm{H}$ 
NMR (500 MHz, $\left.\mathrm{CDCl}_{3}\right) \delta 7.85(\mathrm{dd}, J=1.5,6.5 \mathrm{~Hz}, 2 \mathrm{H}), 7.47-7.40(\mathrm{~m}, 7 \mathrm{H}), 7.27-7.25(\mathrm{~m}, 2 \mathrm{H})$, 7.24-7.18 (m, $1 \mathrm{H}), 7.08-7.06$ (m, $6 \mathrm{H}), 6.87$ (t, $J=7.0 \mathrm{~Hz}, 1 \mathrm{H}), 3.82$ (t, $J=11 \mathrm{~Hz}, 2 \mathrm{H}), 3.49$ (m, 2 H), 3.16-3.06 (m, $2 \mathrm{H}), 2.91-2.81(\mathrm{~m}, 4 \mathrm{H}), 2.18-2.11$ (m, $2 \mathrm{H}), 1.61(\mathrm{~s}, 9 \mathrm{H}) ;{ }^{13} \mathrm{C}$ NMR (100 MHz, $\left.\mathrm{CDCl}_{3}\right) \delta 165.9,146.9,145.2,140.2,138.7,130.10,130.06,129.96,129.86,129.4,129.3,128.7,128.6$, 127.6, 126.3, 117.8, 113.7, 81.0, 63.1, 55.7, 55.5, 54.3, 54.1, 37.6, 37.4, 28.4; IR (film) 2975, 1711, $1598 \mathrm{~cm}^{-1}$; MS (ESI) $533.3166\left(533.3168\right.$ calcd for $\left.\mathrm{C}_{36} \mathrm{H}_{40} \mathrm{~N}_{2} \mathrm{O}_{2}, \mathrm{M}+\mathrm{H}^{+}\right)$.

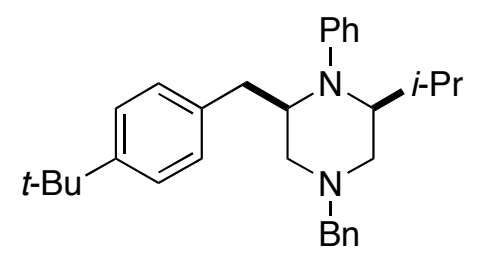

14

(2S,6R)-4-Benzyl-2-(4-tert-butylbenzyl)-6-isopropyl-1-phenylpiperazine (14). The reaction of 150 $\mathrm{mg}(0.49 \mathrm{mmol})$ of $N^{1}$-allyl- $N^{1}$-benzyl-3-methyl- $N^{2}$-phenylbutane-1,2-diamine (1b) with $145 \mathrm{mg}(0.68$ $\mathrm{mmol}$ ) of 4-bromo-tert-butylbenzene was conducted for $10 \mathrm{~h}$ according to the general procedure using 2 mol $\% \mathrm{Pd}_{2}(\mathrm{dba})_{3}$ and $16 \mathrm{~mol} \% \mathrm{P}(2 \text {-furyl })_{3}$. The product was formed with $>20: 1 \mathrm{dr}$ as judged by ${ }^{1} \mathrm{H}$ NMR analysis of a sample taken from the crude reaction mixture. Upon purification, $110 \mathrm{mg}(51 \%)$ of the title compound was obtained as a white solid, m.p. $83-86{ }^{\circ} \mathrm{C}$. This material was judged to be of $>20: 1 \mathrm{dr}$ by ${ }^{1} \mathrm{H}$ NMR analysis. The enantiopurity was judged to be $99 \%$ ee by chiral hplc analysis (chiralcel OD-H column, $100 \%$ hexanes, $1 \mathrm{~mL} / \mathrm{min}, \mathrm{RT}=6.29 \mathrm{~min}$ and $7.06 \mathrm{~min}),[\alpha]^{23}{ }_{\mathrm{D}}+3.13^{\circ}(c$ 0.17, $\left.\mathrm{CH}_{2} \mathrm{Cl}_{2}\right) .{ }^{1} \mathrm{H}$ NMR (400 MHz, $\left.\mathrm{CDCl}_{3}\right) \delta$ 7.36-7.29 (m, $\left.7 \mathrm{H}\right), 7.24-7.18(\mathrm{~m}, 4 \mathrm{H}), 7.01(\mathrm{t}, J=7.2$ $\mathrm{Hz}, 1 \mathrm{H}), 6.95(\mathrm{~d}, J=8.0 \mathrm{~Hz}, 2 \mathrm{H}), 3.57(\mathrm{~d}, J=13.2 \mathrm{~Hz}, 1 \mathrm{H}), 3.49-3.47(\mathrm{~m}, 1 \mathrm{H}), 3.44(\mathrm{~d}, J=16 \mathrm{~Hz}$, $1 \mathrm{H}), 3.30-3.27(\mathrm{~m}, 1 \mathrm{H}), 2.74-2.62(\mathrm{~m}, 2 \mathrm{H}), 2.57-2.54(\mathrm{~m}, 1 \mathrm{H}), 2.49-2.45(\mathrm{~m}, 2 \mathrm{H}), 2.37-2.32(\mathrm{~m}$, $1 \mathrm{H}), 1.94-1.89(\mathrm{~m}, 1 \mathrm{H}), 1.32(9 \mathrm{H}), 0.90(\mathrm{~d}, J=6.8 \mathrm{~Hz}, 3 \mathrm{H}), 0.81(\mathrm{~d}, J=7.2 \mathrm{~Hz}, 3 \mathrm{H}) ;{ }^{13} \mathrm{C} \mathrm{NMR}$ $\left(100 \mathrm{MHz}, \mathrm{CDCl}_{3}\right) \delta 150.3,148.8,138.5,136.8,129.4,129.3,129.0,128.3,127.1,125.2,121.8,63.4$, $63.0,60.7,57.2,53.7,38.3,34.5,31.6,29.8,20.8,18.2$ (one aromatic carbon signal is absent due to incidental equivalence); IR (film) 2915, $1604 \mathrm{~cm}^{-1}$. MS (ESI) 441.3269 (441.3270 calcd for $\mathrm{C}_{31} \mathrm{H}_{40} \mathrm{~N}_{2}$, $\left.\mathrm{M}+\mathrm{H}^{+}\right)$. 


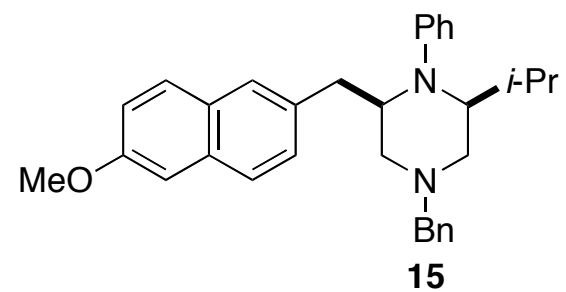

(2S,6R)-4-Benzyl-2-isopropyl-6-(6-methoxynaphthalen-2-ylmethyl)-1-phenylpiperazine (15). The reaction of $100 \mathrm{mg}(0.32 \mathrm{mmol})$ of $N^{1}$-allyl- $N^{1}$-benzyl-3-methyl- $N^{2}$-phenylbutane-1,2-diamine with 108 $\mathrm{mg}(0.45 \mathrm{mmol})$ of 2-bromo-6-methoxynapthalene was conducted for $10 \mathrm{~h}$ according to the general procedure. The product was formed with $>20: 1 \mathrm{dr}$ as judged by ${ }^{1} \mathrm{H}$ NMR analysis of a sample taken from the crude reaction mixture. Upon purification, $77 \mathrm{mg}(51 \%)$ of the title compound was obtained as a white solid, m.p. $114-119{ }^{\circ} \mathrm{C}$. This material was judged to be of $>20: 1 \mathrm{dr}$ by ${ }^{1} \mathrm{H}$ NMR analysis. The enantiopurity was judged to be $99 \%$ ee by chiral hplc analysis (chiralcel AD column, 5\% isopropanol/hexanes, $0.9 \mathrm{~mL} / \mathrm{min}, \mathrm{RT}=4.22 \mathrm{~min}$ and $5.78 \mathrm{~min}),[\alpha]^{23}{ }_{\mathrm{D}}+33.5^{\circ}\left(c 0.34, \mathrm{CH}_{2} \mathrm{Cl}_{2}\right) .{ }^{1} \mathrm{H}$ NMR (400 MHz, $\left.\mathrm{CDCl}_{3}\right) \delta 7.59(\mathrm{dd}, J=6.4,8.8 \mathrm{~Hz}, 2 \mathrm{H}), 7.36-7.26(\mathrm{~m}, 7 \mathrm{H}), 7.21-7.19$ (m, $\left.3 \mathrm{H}\right)$, 7.13-7.07 (m, $3 \mathrm{H}), 7.01(\mathrm{t}, J=7.2 \mathrm{~Hz}, 1 \mathrm{H}), 3.91(\mathrm{~s}, 3 \mathrm{H}), 3.56(\mathrm{~d}, J=12.8 \mathrm{~Hz}, 1 \mathrm{H}), 3.54-3.49(\mathrm{~m}, 1$ H), $3.35(\mathrm{~d}, J=13.2 \mathrm{~Hz}, 1 \mathrm{H}), 3.29-3.25(\mathrm{~m}, 1 \mathrm{H}), 2.86-2.73(\mathrm{~m}, 2 \mathrm{H}), 2.55(\mathrm{dd}, J=3.2,10.8 \mathrm{~Hz}, 1$ H), 2.47-2.41 (m, $2 \mathrm{H}), 2.35$ (dd, $J=6.4,10.8 \mathrm{~Hz}, 1 \mathrm{H}), 1.93-1.87$ (m, $1 \mathrm{H}), 0.89$ (d, $J=6.8 \mathrm{~Hz}, 3 \mathrm{H})$, $0.79(\mathrm{~d}, J=7.2 \mathrm{~Hz}, 3 \mathrm{H}) ;{ }^{13} \mathrm{C}$ NMR $\left(100 \mathrm{MHz}, \mathrm{CDCl}_{3}\right) \delta 157.4,150.4,138.5,135.1,133.2,129.44$, 129.36, 129.17, 129.16, 128.5, 128.4, 127.6, 127.2, 126.8, 122.0, 118.8, 105.8, 63.5, 63.2, 60.8, 57.3, $55.5,53.7,39.0,29.8,20.8,18.2$ (one aromatic carbon signal is absent due to incidental equivalence); IR (film) 2956, $1604 \mathrm{~cm}^{-1}$; MS (ESI) 465.2899 (465.2906 calcd for $\mathrm{C}_{32} \mathrm{H}_{36} \mathrm{~N}_{2} \mathrm{O}, \mathrm{M}+\mathrm{H}^{+}$).

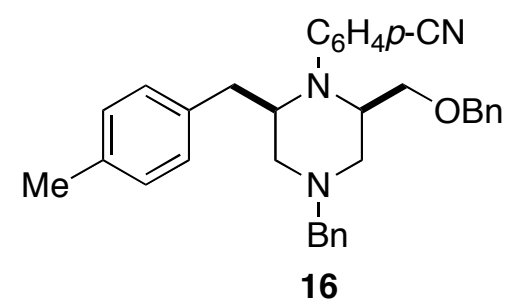

(2R,6R)-4-[4-Benzyl-2-benzyloxymethyl-6-(4-methylbenzyl)piperazin-1-yl]benzonitrile (16). The reaction of $150 \mathrm{mg} \quad(0.37 \mathrm{mmol})$ of $(R)-4-\{1$-[allyl(benzyl)amino]-3-(benzyloxy)propan-2ylamino\}benzonitrile (1c) with $75 \mathrm{mg}(0.48 \mathrm{mmol})$ of 4-bromotoluene was conducted for $10 \mathrm{~h}$ according to the general procedure. The product was formed with 14:1 dr as judged by ${ }^{1} \mathrm{H}$ NMR analysis of a sample taken from the crude reaction mixture. Upon purification, $128 \mathrm{mg}(70 \%)$ of the title compound was obtained as a white solid, m.p. $125-133{ }^{\circ} \mathrm{C}$. This material was determined to 
contain a 14:1 mixture of diastereomers as judged by ${ }^{1} \mathrm{H}$ NMR analysis. The enantiopurity of the major diastereomer was judged to be $97 \%$ ee by chiral hplc analysis (chiralcel AD column, $10 \%$ isopropanol/hexanes, $1 \mathrm{~mL} / \mathrm{min}, \mathrm{RT}=5.32 \mathrm{~min}$ and $7.41 \mathrm{~min}),[\alpha]^{23}{ }_{\mathrm{D}}+168.99^{\circ}\left(c 0.17, \mathrm{CH}_{2} \mathrm{Cl}_{2}\right)$. Data are for the major diastereomer: ${ }^{1} \mathrm{H}$ NMR $\left(500 \mathrm{MHz}, \mathrm{CDCl}_{3}\right) \delta 7.53(\mathrm{~d}, J=9.0 \mathrm{~Hz}, 2 \mathrm{H}), 7.40-7.33(\mathrm{~m}$, 7 H), 7.32-7.28 (m, $3 \mathrm{H}), 6.96(\mathrm{~d}, J=8.0 \mathrm{~Hz}, 2 \mathrm{H}), 6.84(\mathrm{~d}, J=9.0 \mathrm{~Hz}, 2 \mathrm{H}), 6.69(\mathrm{~d}, J=8.0 \mathrm{~Hz}, 2 \mathrm{H})$, 4.55-4.48 (m, 2 H), 3.95-3.89 (m, 2 H), 3.72-3.70 (m, 1 H), 3.61 (d, J = 12 Hz, $1 \mathrm{H}), 3.39-3.35$ (m, 2 H), $3.26(\mathrm{dd}, J=1.5,11 \mathrm{~Hz}, 1 \mathrm{H}), 2.83(\mathrm{~d}, J=11.5 \mathrm{~Hz}, 1 \mathrm{H}), 2.75(\mathrm{t}, J=12 \mathrm{~Hz}, 1 \mathrm{H}), 2.44(\mathrm{~d}, J=12.5$ $\mathrm{Hz}, 1 \mathrm{H}), 2.31-2.28(\mathrm{M}, 1 \mathrm{H}), 2.28(\mathrm{~s}, 3 \mathrm{H}), 1.96(\mathrm{dd}, J=3.0,11 \mathrm{~Hz}, 1 \mathrm{H}) ;{ }^{13} \mathrm{C}$ NMR $(125 \mathrm{MHz}$, $\left.\mathrm{CDCl}_{3}\right) \delta$ 150.2, 138.6, 138.2, 136.2, 135.8, 134.1, 129.8, 129.5, 129.2, 128.7, 128.6, 128.13, 128.06, 127.6, 120.6, 112.1, 99.6, 73.7, 68.5, 62.8, 55.0, 54.4, 53.1, 52.6, 37.0, 21.2; IR (film) 2919, $1603 \mathrm{~cm}^{-1}$; MS (ESI) $524.2679\left(524.2678\right.$ calcd for $\left.\mathrm{C}_{34} \mathrm{H}_{35} \mathrm{~N}_{3} \mathrm{O}, \mathrm{M}+\mathrm{Na}^{+}\right)$.

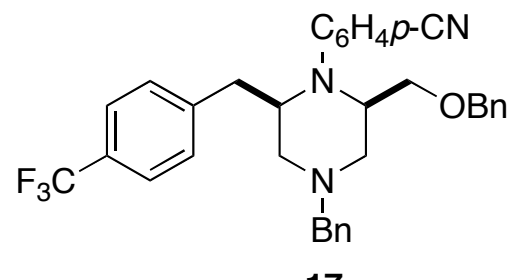

17

$(2 R, 6 R)$-4-[4-Benzyl-2-benzyloxymethyl-6-(4-trifluoromethylbenzyl)piperazin-1-yl]benzonitrile

(17). The reaction of $150 \mathrm{mg}(0.37 \mathrm{mmol})$ of $(R)-4$-\{1-[allyl(benzyl)amino]-3-(benzyloxy)propan-2ylamino\}benzonitrile (1c) with $98 \mathrm{mg}(0.44 \mathrm{mmol})$ of 4-bromobenzotrifluoride was conducted for $10 \mathrm{~h}$ according to the general procedure. The product was formed with 14:1 dr as judged by ${ }^{1} \mathrm{H}$ NMR analysis of a sample taken from the crude reaction mixture. Upon purification, $131 \mathrm{mg}(65 \%)$ of the title compound was obtained as a white solid, m.p. $154-157^{\circ} \mathrm{C}$. This material was determined to contain a 14:1 mixture of diastereomers as judged by ${ }^{1} \mathrm{H}$ NMR analysis. The enantiopurity of the major diastereomer was judged to be $99 \%$ ee by chiral hplc analysis (chiralcel AD column, $10 \%$ isopropanol/hexanes, $1 \mathrm{~mL} / \mathrm{min}, \mathrm{RT}=5.99 \mathrm{~min}$ and $8.68 \mathrm{~min}),[\alpha]^{23}{ }_{\mathrm{D}}+155.58^{\circ}\left(c 0.13, \mathrm{CH}_{2} \mathrm{Cl}_{2}\right)$. The diastereomers were subsequently separated by careful flash chromatography on silica gel.

Major (cis) diastereomer (17): ${ }^{1} \mathrm{H}$ NMR (400 MHz, $\left.\mathrm{CDCl}_{3}\right) \delta 7.54$ (d, $\left.J=8.0 \mathrm{~Hz}, 2 \mathrm{H}\right), 7.41-7.31(\mathrm{~m}$, $12 \mathrm{H}), 6.82(\mathrm{t}, J=8.8 \mathrm{~Hz}, 4 \mathrm{H}), 4.59-4.51(\mathrm{~m}, 2 \mathrm{H}), 3.97-3.89$ (m, $2 \mathrm{H}), 3.74-3.71$ (m, $1 \mathrm{H}), 3.66$ (d, $J$ $=12.4 \mathrm{~Hz}, 1 \mathrm{H}), 3.38(\mathrm{~d}, J=6.8 \mathrm{~Hz}, 1 \mathrm{H}), 3.32-3.27(\mathrm{~m}, 2 \mathrm{H}), 2.80(\mathrm{t}, J=12.4 \mathrm{~Hz}, 1 \mathrm{H}), 2.71(\mathrm{~d}, J=$ $11.6,1 \mathrm{H}), 2.51(\mathrm{~d}, J=12.4 \mathrm{~Hz}, 1 \mathrm{H}), 2.37(\mathrm{dd}, J=2.4,11.6 \mathrm{~Hz}, 1 \mathrm{H}), 1.95(\mathrm{dd}, J=3.2,12 \mathrm{~Hz}, 1 \mathrm{H})$; ${ }^{13} \mathrm{C}$ NMR (500 MHz, $\mathrm{CDCl}_{3}$ ) $\delta 150.0,142.8,138.4,138.1,134.1,130.0,129.6,129.0,128.74,128.69$, 
128.2, 128.1, 127.7, $125.6(\mathrm{q}, J=14.5 \mathrm{~Hz}), 124.3(\mathrm{q}, J=270.5 \mathrm{~Hz}), 120.4,112.1,99.1,73.6,68.4$, 62.8, 54.7, 54.5, 53.2, 52.1, 37.3; IR (film) 2817, $1603 \mathrm{~cm}^{-1}$; MS (ESI) 578.2402 (578.2395 calcd for $\left.\mathrm{C}_{34} \mathrm{H}_{32} \mathrm{~F}_{3} \mathrm{~N}_{3} \mathrm{O}, \mathrm{M}+\mathrm{Na}^{+}\right)$.

Minor (trans) diastereomer (17b): ${ }^{1} \mathrm{H}$ NMR $\left(400 \mathrm{MHz}, \mathrm{CDCl}_{3}\right) \delta 7.54$ (d, $\left.J=8.4 \mathrm{~Hz}, 2 \mathrm{H}\right), 7.37-7.26$ (m, $11 \mathrm{H}), 7.19-7.17(\mathrm{~m}, 2 \mathrm{H}), 7.10(\mathrm{~d}, J=8.4 \mathrm{~Hz}, 2 \mathrm{H}), 6.79(\mathrm{~d}, J=8.0 \mathrm{~Hz}, 2 \mathrm{H}), 4.38(\mathrm{~s}, 2 \mathrm{H}), 3.80-$ $3.76(\mathrm{~m}, 1 \mathrm{H}), 3.68-3.62(\mathrm{~m}, 2 \mathrm{H}), 3.49(\mathrm{dd}, J=2.4,9.6 \mathrm{~Hz}, 1 \mathrm{H}), 3.34-3.31(\mathrm{~m}, 2 \mathrm{H}), 3.19(\mathrm{dd}, J=$ 2.4, $11.2 \mathrm{~Hz}, 1 \mathrm{H}), 3.03-2.97$ (m, $1 \mathrm{H}), 2.53-2.49$ (m, $2 \mathrm{H}), 2.46-2.41$ (m, $1 \mathrm{H}), 2.30$ (dd, $J=3.2,11.2$ $\mathrm{Hz}, 1 \mathrm{H})$.

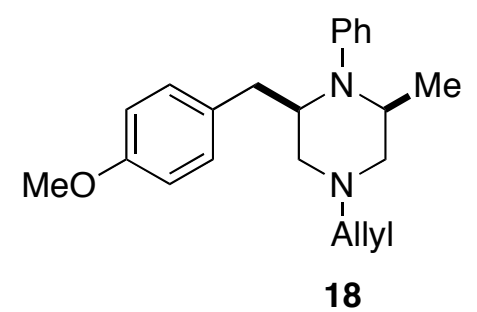

(2R,6S)-4-Allyl-2-(4-methoxybenzyl)-6-methyl-1-phenylpiperazine (18). The reaction of $124 \mathrm{mg}$ $(0.54 \mathrm{mmol})$ of $(S)-N^{1}, N^{1}$-diallyl- $N^{2}$-phenylpropane-1,2-diamine (1d) with $121 \mathrm{mg}(0.65 \mathrm{mmol})$ of 4 bromoanisole was conducted for $10 \mathrm{~h}$ according to the general procedure. The product was formed with $>20: 1 \mathrm{dr}$ as judged by ${ }^{1} \mathrm{H}$ NMR analysis of a sample taken from the crude reaction mixture. Upon purification, $91 \mathrm{mg} \mathrm{(50 \% )} \mathrm{of} \mathrm{the} \mathrm{title} \mathrm{compound} \mathrm{was} \mathrm{obtained} \mathrm{as} \mathrm{a} \mathrm{yellow} \mathrm{oil.} \mathrm{This} \mathrm{material} \mathrm{was} \mathrm{judged}$ to be of $>20: 1 \mathrm{dr}$ by ${ }^{1} \mathrm{H}$ NMR analysis. The enantiopurity was judged to be $99 \%$ ee by chiral hplc analysis (OD-H column, $100 \%$ hexanes, $1 \mathrm{~mL} / \mathrm{min}, \mathrm{RT}=19.75 \mathrm{~min}$ and $22.39 \mathrm{~min}),[\alpha]^{23}{ }_{\mathrm{D}}+112.18(c$ $0.26, \mathrm{CH}_{2} \mathrm{Cl}_{2}$ ). ${ }^{1} \mathrm{H}$ NMR (500 MHz, $\left.\mathrm{CDCl}_{3}\right) \delta 7.35(\mathrm{t}, J=8.5 \mathrm{~Hz}, 2 \mathrm{H}), 7.17(\mathrm{~d}, J=8.0 \mathrm{~Hz}, 2 \mathrm{H}), 7.07$ $(\mathrm{t}, J=7.5 \mathrm{~Hz}, 1 \mathrm{H}), 6.98(\mathrm{~d}, J=9.0 \mathrm{~Hz}, 2 \mathrm{H}), 6.79-6.76(\mathrm{~m}, 2 \mathrm{H}), 5.90-5.82(\mathrm{~m}, 1 \mathrm{H}), 5.20-5.12(\mathrm{~m}, 2$ H), $3.77(\mathrm{~s}, 3 \mathrm{H}), 3.45-3.40(\mathrm{~m}, 1 \mathrm{H}), 3.38-3.35(\mathrm{~m}, 1 \mathrm{H}), 3.06-3.01(\mathrm{~m}, 1 \mathrm{H}), 2.92-2.88(\mathrm{~m}, 1 \mathrm{H})$, $2.71(\mathrm{dd}, J=2.0,11 \mathrm{~Hz}, 1 \mathrm{H}), 2.57(\mathrm{dd}, J=3.5,13.5 \mathrm{~Hz}, 1 \mathrm{H}), 2.51(\mathrm{~d}, J=10 \mathrm{~Hz}, 1 \mathrm{H}), 2.44-2.40(\mathrm{~m}$, $1 \mathrm{H}), 2.26-2.18(\mathrm{~m}, 2 \mathrm{H}), 0.94(\mathrm{~d}, J=6.5 \mathrm{~Hz}, 3 \mathrm{H}) ;{ }^{13} \mathrm{C} \mathrm{NMR}\left(100 \mathrm{MHz}, \mathrm{CDCl}_{3}\right) \delta 158.1,149.2$, 135.2, 131.8, 130.2, 129.4, 123.2, 118.1, 113.8, 61.9, 60.5, 60.4, 57.4, 55.4, 54.2, 37.9, 18.8 (one carbon signal is absent due to incidental equivalence); IR (film) 3400, 2929, $1597 \mathrm{~cm}^{-1}$; MS (ESI) 337.2263 (337.2280 calcd for $\mathrm{C}_{22} \mathrm{H}_{28} \mathrm{~N}_{2} \mathrm{O}, \mathrm{M}+\mathrm{H}^{+}$). 


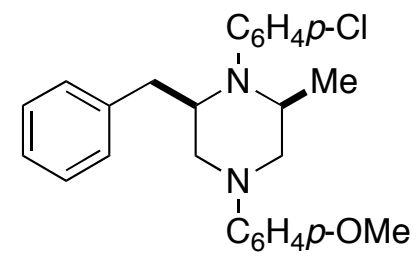

19

(2R,6S)-2-Benzyl-1-(4-chlorophenyl)-4-(4-methoxyphenyl)-6-methylpiperazine (19). The reaction of $47 \mathrm{mg}(0.14 \mathrm{mmol})$ of $N^{1}$-allyl- $N^{2}$-(4-chlorophenyl)- $N^{1}$-(4-methoxyphenyl)propane-1,2-diamine (1e) with $22 \mathrm{mg}(0.17 \mathrm{mmol})$ of bromobenzene was conducted for $10 \mathrm{~h}$ according to the general procedure. The product was formed with $>20: 1 \mathrm{dr}$ as judged by ${ }^{1} \mathrm{H}$ NMR analysis of a sample taken from the crude reaction mixture. Upon purification, $29 \mathrm{mg}(51 \%)$ of the title compound was obtained as a yellow oil. This material was judged to be of $>20: 1 \mathrm{dr}$ by ${ }^{1} \mathrm{H}$ NMR analysis. The enantiopurity was judged to be $99 \%$ ee by chiral hplc analysis (chiralcel OD-H column, 1\% isopropanol/hexanes, 0.5 $\mathrm{mL} / \mathrm{min}, \mathrm{RT}=9.27 \mathrm{~min}$ and $10.30 \mathrm{~min}),[\alpha]^{23}{ }_{\mathrm{D}}+58.87^{\circ}\left(c 0.55, \mathrm{CH}_{2} \mathrm{Cl}_{2}\right) .{ }^{1} \mathrm{H} \mathrm{NMR}\left(500 \mathrm{MHz}, \mathrm{CDCl}_{3}\right)$

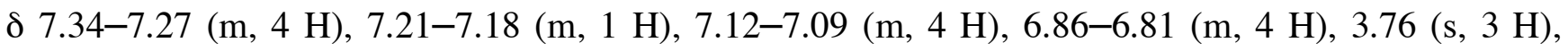
3.59-3.55 (m, $1 \mathrm{H}), 3.52-3.49(\mathrm{~m}, 1 \mathrm{H}), 3.25(\mathrm{dd}, J=3.5,12 \mathrm{~Hz}, 1 \mathrm{H}), 3.07(\mathrm{dd}, J=3.0,12 \mathrm{~Hz}, 1 \mathrm{H})$, 2.92-2.88 (m, $2 \mathrm{H}), 2.68(\mathrm{dd}, J=3.5,13.5 \mathrm{~Hz}, 1 \mathrm{H}), 2.58(\mathrm{dd}, J=10.5,13.5 \mathrm{~Hz}, 1 \mathrm{H}), 1.05$ (d, $J=6.5$ $\mathrm{Hz}, 3 \mathrm{H}) ;{ }^{13} \mathrm{C} \mathrm{NMR}\left(125 \mathrm{MHz}, \mathrm{CDCl}_{3}\right) \delta 154.2,147.5,146.0,139.4,129.5,129.3,128.7,128.0,126.5$, 123.6, 118.9, 114.7, 60.2, 58.3, 55.8, 54.7, 53.8, 38.6, 18.5; IR (film) 2930, $1510 \mathrm{~cm}^{-1}$; MS (ESI) 407.1895 (407.1890 calcd for $\mathrm{C}_{25} \mathrm{H}_{27} \mathrm{ClN}_{2} \mathrm{O}, \mathrm{M}+\mathrm{H}^{+}$).

\section{Isolation and Characterization of Side Products 10 and 11}

Side products 10 and 11 were isolated by careful chromatography of the crude mixture of products obtained from the $\mathrm{Pd}_{2}(\mathrm{dba})_{3} / \mathrm{P}(o \text {-tol })_{3}$-catalyzed reaction of $\mathbf{1 a}$ with 4-bromoanisole. These compounds were characterized by ${ }^{1} \mathrm{H}$ NMR and 2-D COSY analysis. Data are as follows:

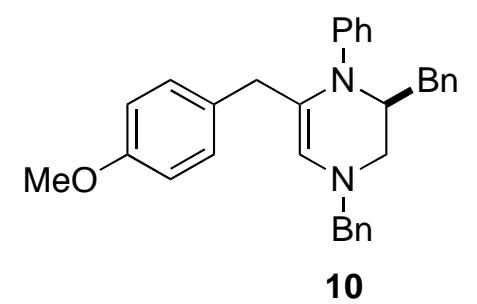

(S)-4-allyl-6-(4-methoxybenzyl)-2-methyl-1-phenyl-1,2,3,4-tetrahydropyrazine (10). ${ }^{1} \mathrm{H}$ NMR (400 $\left.\mathrm{MHz}, \mathrm{CDCl}_{3}\right) \delta$ 7.33-7.31 (m, $\left.4 \mathrm{H}\right), 7.20-7.12(\mathrm{~m}, 5 \mathrm{H}), 6.99-6.97(\mathrm{~m}, 2 \mathrm{H}), 6.89-6.85(\mathrm{~m}, 1 \mathrm{H})$, 
6.80-6.77 (m, $2 \mathrm{H}), 6.75-6.73(\mathrm{~m}, 2 \mathrm{H}), 6.69(\mathrm{~d}, J=8.0 \mathrm{~Hz}, 1 \mathrm{H}), 6.60(\mathrm{dd}, J=1.0,8.5 \mathrm{~Hz}, 2 \mathrm{H}), 5.82$ (s, $1 \mathrm{H}), 4.08-3.98(\mathrm{~m}, 2 \mathrm{H}), 3.79(\mathrm{~s}, 3 \mathrm{H}), 3.56-3.52(\mathrm{~m}, 1 \mathrm{H}), 3.36(\mathrm{~d}, J=10.5,15.5 \mathrm{~Hz}, 1 \mathrm{H}), 3.14$ $(\mathrm{d}, J=15 \mathrm{~Hz}, 1 \mathrm{H}), 2.74-2.66(\mathrm{~m}, 2 \mathrm{H}), 2.61-2.59(\mathrm{~m}, 1 \mathrm{H}), 2.49(\mathrm{dd}, J=5.0,13 \mathrm{~Hz}, 1 \mathrm{H})$.

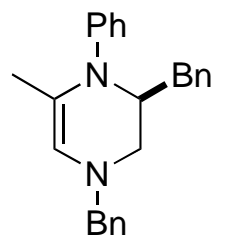

11

(S)-4-allyl-2,6-dimethyl-1-phenyl-1,2,3,4-tetrahydropyrazine (11). ${ }^{1} \mathrm{H} \mathrm{NMR}\left(500 \mathrm{MHz}, \mathrm{CDCl}_{3}\right) \delta$ 7.34-7.19 (m, $10 \mathrm{H}), 7.13-7.10$ (m, 2 H), 6.82 (dt, J=1.0, 7.0 Hz, $1 \mathrm{H}), 6.56$ (d, J = 8.0 Hz, 2 H), 5.68 (s, $1 \mathrm{H}), 4.04-3.95(\mathrm{~m}, 2 \mathrm{H}), 3.67-3.64(\mathrm{~m}, 1 \mathrm{H}), 3.07(\mathrm{dd}, J=4.0,13 \mathrm{~Hz}, 1 \mathrm{H}), 2.73-2.68(\mathrm{~m}, 2 \mathrm{H})$, $2.62(\mathrm{dd}, J=2.5,10.5 \mathrm{~Hz}, 1 \mathrm{H}), 1.73(\mathrm{~s}, 3 \mathrm{H})$.

\section{Assignment of Stereochemistry}

\section{2-Isopropylpiperazines 14 and 15}

The stereochemistry of 2-isopropylpiperazine 15 was assigned on the basis of 2D NOESY experiments. The key nOe signals are shown below. The stereochemistry of piperazine $\mathbf{1 4}$ was assigned based on analogy to $\mathbf{1 5 .}$

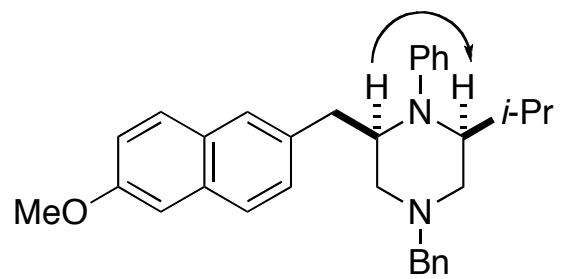

15

\section{2-Benzyloxymethylpiperazines 16 and 17}

The stereochemistry of 2-benzyloxymethylpiperazine 17 was assigned on the basis of 2D NOESY experiments. The key nOe signals are shown below. The stereochemistry of piperazine $\mathbf{1 6}$ was assigned based on analogy to 17 . 


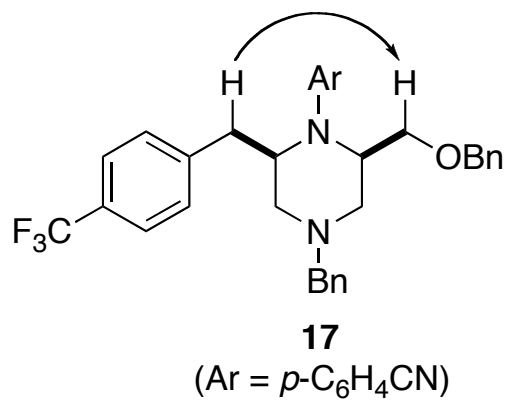

\section{2-Methylpiperazine 19}

The stereochemistry of 2-methylpiperazine 19 was assigned on the basis of 2D NOESY experiments. The key nOe signals are shown below.

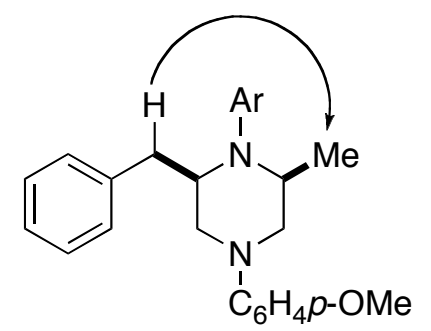

19

$\left(\mathrm{Ar}=\mathrm{C}_{6} \mathrm{H}_{4} \mathrm{Cl}\right)$

\section{2-Methylpiperazine 18 and 2-Benzylpiperazines 9, 12, and 13}

The stereochemistry of 2-methylpiperazine $\mathbf{1 8}$ was assigned on the basis of 2D NOESY experiments. The key nOe signals are shown below. The stereochemistry of 2-benzylpiperazines $\mathbf{9 , 1 2}$, and $\mathbf{1 3}$ was assigned based on analogy to $\mathbf{1 7}$.

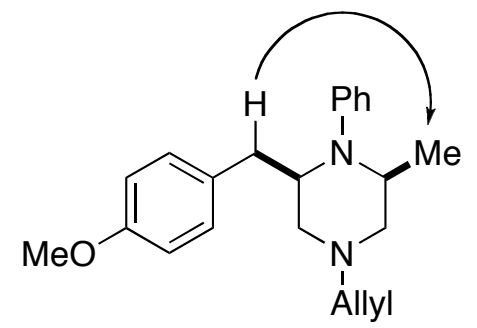

18

\section{References}

(1) Ma, D.; Zhang, Y.; Yao, J.; Wu, S.; Tao, F. J. Am. Chem. Soc. 1998, 120, 12459-12467.

(2) Li, H.; Jiang, X.; Ye, Y.-h.; Fan, C.; Romoff, T.; Goodman, M. Org. Lett. 1999, 1, 91-94.

(3) Cai,Q.; Zhu, W.; Zhang, H.; Zhang, Y.; Ma, D. Synthesis 2005, 496-499. 